\title{
Whole genome re-sequencing of sweet cherry (Prunus avium L.) yields insights into genomic diversity of a fruit species
}

\author{
Aliki Xanthopoulou', Maria Manioudaki', Christos Bazakos², Christos Kissoudis ${ }^{3}$, Anna-Maria Farsakoglou ${ }^{4}$, \\ Evangelos Karagiannis ${ }^{1}$, Michail Michailidis ${ }^{1}$, Chrysanthi Polychroniadou' ${ }^{1}$ Antonios Zambounis ${ }^{5}$, \\ Konstantinos Kazantzis ${ }^{5}$, Athanasios Tsaftaris ${ }^{3}$, Panagiotis Madesis ${ }^{6}$, Filippos Aravanopoulos, \\ Athanassios Molassiotis (D) ${ }^{1}$ and loannis Ganopoulos ${ }^{2}$
}

\begin{abstract}
Sweet cherries, Prunus avium L. (Rosaceae), are gaining importance due to their perenniallity and nutritional attributes beneficial for human health. Interestingly, sweet cherry cultivars exhibit a wide range of phenotypic diversity in important agronomic traits, such as flowering time and defense reactions against pathogens. In this study, wholegenome resequencing (WGRS) was employed to characterize genetic variation, population structure and allelic variants in a panel of 20 sweet cherry and one wild cherry genotypes, embodying the majority of cultivated Greek germplasm and a representative of a local wild cherry elite phenotype. The 21 genotypes were sequenced in an average depth of coverage of 33.91×. and effective mapping depth, to the genomic reference sequence of 'Satonishiki' cultivar, between $22.21 \times$ to $36.62 \times$. Discriminant analysis of principal components (DAPC) with SNPs revealed two clusters of genotypes. There was a rapid linkage disequilibrium decay, as the majority of SNP pairs with $r^{2}$ in near complete disequilibrium ( $>0.8$ ) were found at physical distances less than $10 \mathrm{~kb}$. Functional analysis of the variants showed that the genomic ratio of non-synonymous/synonymous (dN/dS) changes was 1.78. The higher $d N$ frequency in the Greek cohort of sweet cherry could be the result of artificial selection pressure imposed by breeding, in combination with the vegetative propagation of domesticated cultivars through grafting. The majority of SNPs with high impact (e.g., stop codon gaining, frameshift), were identified in genes involved in flowering time, dormancy and defense reactions against pathogens, providing promising resources for future breeding programs. Our study has established the foundation for further large scale characterization of sweet cherry germplasm, enabling breeders to incorporate diverse germplasm and allelic variants to fine tune flowering and maturity time and disease resistance in sweet cherry cultivars.
\end{abstract}

\section{Introduction}

Prunus avium L. (Rosaceae), is a fruit crop with growing agronomic and economical importance. For instance, in

\footnotetext{
Correspondence: Aliki Xanthopoulou (aliki.xanthopoulou@gmail.com) or loannis Ganopoulos (giannis.ganopoulos@gmail.com)

'Laboratory of Pomology, Department of Agriculture, Aristotle University of Thessaloniki, 54124 Thessaloniki, Greece

${ }^{2}$ Institute of Plant Breeding and Genetic Resources, ELGO-DEMETER. Thermi, Thessaloniki 570001, Greece

Full list of author information is available at the end of the article
}

the past decade, production acreage of sweet cherries in Greece has been increased by 4000 ha, representing more than a $50 \%$ increase, while production has also increased by roughly the same percentage to an average of 73,000 tons per year in 2016 (FAOSTAT, 2016) (http://www.fao. org/faostat/en/?\#data/QC/visualize). Sweet cherry consumption has potential preventative benefits against (among others) Alzheimer's, cancer, and inflammation related diseases ${ }^{1}$. Due to the economic importance of

\section{(c) The Author(s) 2020}

\footnotetext{
(cc) Open Access This article is licensed under a Creative Commons Attribution 4.0 International License, which permits use, sharing, adaptation, distribution and reproduction c. in any medium or format, as long as you give appropriate credit to the original author(s) and the source, provide a link to the Creative Commons license, and indicate if changes were made. The images or other third party material in this article are included in the article's Creative Commons license, unless indicated otherwise in a credit line to the material. If material is not included in the article's Creative Commons license and your intended use is not permitted by statutory regulation or exceeds the permitted use, you will need to obtain permission directly from the copyright holder. To view a copy of this license, visit http://creativecommons.org/licenses/by/4.0/.
} 
sweet cherry, its potential benefit for human health and its advancing position in Greek agriculture, it is vital to provide research support that would maintain global competitiveness for local growers ${ }^{2}$.

Sweet cherries exhibit important phenotypic variation in fruit size, shape, color, sugar content, flowering time and other agronomic traits such as defense reactions against pathogens ${ }^{3}$. The reason for the development of genetic variability in sweet cherry cultivars which originate from Black and Caspian Seas is their adaptation in the context of spreading. The deeper study and characterization of sweet cherry genetic diversity as well as the identification of genes controlling traits of interest will be a key factor for sweet cherry breeding. Greece contains high rates of genetic diversity, although many of local traditional landraces have been lost over years of evolution $^{2}$. Moreover, in various other countries, a narrow genetic bottleneck has been observed, in modern cultivars ${ }^{4}$. In Greece, various studies of molecular diversity, of modern cherry varieties, have been conducted using SSR markers, revealing their extensive genetic basis, a valuable finding that has been extensively used in breeding programs $^{2,5}$. Genomic characterization of sweet cherry germplasm, has been enriched with tools, such as the sweet and sour cherry $6 \mathrm{~K}$ array $^{6}$. Whole Genome ReSequencing and other such techniques are the springboard for the discovery of allelic and genomic richness.

Identifying allelic variation has gone a step further with the introduction of re-sequencing strategies. Deeper sequencing of genotypes of numerous species has been achieved with the use of high-throughput Next-Generation Sequencing technologies for genome-wide analysis. The genetic background of various fruit-tree species, including citrus ${ }^{7}$, plum $^{8}$, mei $^{9}$, and peach ${ }^{10}$ has been studied with the recently developed, Whole-genome resequencing (WGRS) technique. In order to better understand the genetic basis of plant variation and to incorporate this knowledge into breeding programs, the exploitation of valuable sequences continuously provided in public databases, is of the utmost importance.

The genetic basis of phenotypic diversity has become even more enlightened by the use of genomic resources, such as the development of single nucleotide polymorphism (SNP) maps of genomes. Furthermore, a variety of polymorphisms in underlying loci has been recognized $^{11}$. Perennials, including fruit trees, have been studied to a much smaller scale compared to annual plants. In order to evolve breeding and ensure global food security, the exploitation of genomic resources and phenotypic diversity of perennial taxa is of paramount importance $^{12}$.

The recent release of the first version of sweet cherry genome assembly of 'Satonishiki' cultivar by Shirasawa et al. ${ }^{13}$ in conjunction with the release of chloroplast and mitochondrial genome sequences ${ }^{14,15}$ have enabled new discoveries, including the identification of the SI locus ${ }^{16}$.

In this article, we present analyses of whole genome resequencing of 20 sweet-cherry cultivars and one wildcherry genotype. The 20 Greek genotypes span the traditional range of sweet cherry cultivation and represent the majority of variation (>95\%) in Greece $^{2}$ while this collection is complemented by the presence of an elite local wild cherry genotype. The sequence analysis focused on genomic regions associated with propitious variation, such as deletions, substitutions and duplications, providing the first comprehensive catalog of molecular variation in this species, which could be helpful for explaining divergence/similarity among different variants.

\section{Materials and methods \\ Plant material}

Twenty cultivated sweet-cherry genotypes were selected (Supplementary File 1) from the Greek Fruit Gene Bank collection in Naousa (Institute of Plant Breeding and Genetic Resources-H.A.O. ELGO DEMETER), in Greece, to represent the total diversity of Greek sweet-cherry cultivars $^{2}$. The elite wild cherry genotype was obtained from the Wild Cherry Gene Bank, located in the Xyloupolis Forest Nursery of the Hellenic Forest Service in Greece. The 20 sweet cherry accessions are traditional Greek cultivars, whereas the wild cherry one, was used as an outgroup. We predefined four groups as 'Breeding line', 'Landrace', 'Modern cultivar' and 'Wild' (Supplementary File 1). The morpho-physiological characterization of these twenty sweet cherry cultivars, has been described previously ${ }^{17}$. Genomic DNA was isolated from a pool of young leaf samples of five plants per genotype using NucleoSpin Plant II kit (Macherey-Nagel).

\section{Library construction and whole genome re-sequencing}

Isolated DNA was fragmented with Bioruptor (ThermoFisher Scientific, Waltham, MA, USA) to generate of approximately $300 \mathrm{bp}$ library insert size. Quantity and quality control of the libraries were carried out with Qubit dsDNA HS Assay kit (ThermoFisher Scientific) and Agilent 2100 Bioanalyzer System (Agilent Technologies, Santa Clara, CA, USA), respectively. Highquality DNA libraries were sequenced with the BGISEQ-500 platform (BGI-Tianjin) with read lengths of $100 \mathrm{bp}$.

\section{Reads pre-processing}

The raw sequencing data generated from the BGI platform were filtered for adapter contamination, lowquality reads, duplicated reads and short reads (length < $35 \mathrm{bp}$ ). This filtering produced the high quality 'final raw data' with an average sequencing depth of 33.91x. 


\section{Read mapping, SNP calling and annotation}

The high quality paired-end sequencing reads (100 bp) from each genotype were mapped to the $P$. avium genome (version 1.0.a1) (https://www.rosaceae.org/ species/prunus_avium/genome_v1.0.a1) reference genome using BWA (v0.7.12) ${ }^{18}$. Then the Sequence Alignment/Map (SAM) files were sorted using the Picard software (http://picard.sourceforge.net). GATK genome analysis toolkit (version 4.1.4.1; https://hub.docker.com/ $\mathrm{r}$ /broadinstitute/gatk/) was employed to call SNPs and InDels across all 21 genotypes simultaneously by using standard hard filtering parameters of HaplotypeCaller and SelectVariant, respectively ${ }^{19}$.

SNP annotation was performed based on the P. avium Genome v1.0.a1 using snpEff software ${ }^{20}$, and SNPs were categorized into intergenic upstream or downstream regions and introns or exons. SNPs in coding exons were further classified as synonymous or non-synonymous SNPs. InDels in exons were grouped according to whether they occur a frameshift.

\section{Genetic diversity and population structure}

The 21 genotypes were categorized in 4 groups "Wild", "Landraces", "Breeding lines" and "Modern cultivars" (Supplementary File 1). Nucleotide diversity $(\pi)$ and Tajima's D of each group and population-divergence (Fixation index, $\mathrm{F}_{\mathrm{ST}}$ ) between each group were calculated by using VCFtools (v0.1.14) with a $20 \mathrm{~kb}$ sliding window across the P. avium Genome v1.0.a1 reference genome.

Population genetic structure was assessed using the following methods. Initially, Nei's distance was calculated and a neighbor-joining dendrogram of the 21 genotypes was build using StAMPP ${ }^{21}$ and $a p e^{22} \mathrm{R}$ packages. Then, clustering of the 21 genotypes was performed by using the Discriminant Analysis of Principal Components (DAPC) implemented to the R package, Adegenet ${ }^{23}$. The optimal number of clusters was calculated using the Bayesian Information Criterion (BIC) as described by Jombard et al. ${ }^{23}$. Lastly, gene flow among populations was estimated by calculating the $\mathrm{F}_{\mathrm{ST}}$ for pairwise comparisons for all populations using the StAMPP package in $R$ and hierarchical clustering using the ward.d2 method $^{24}$.

\section{Structural variant discovery and annotation}

BreakDancer (http://breakdancer.sourceforge.net/), SOAPcnv $^{25}$, DELLY ${ }^{26}$ and LUMPY ${ }^{27}$ were used for structure variants (SVs) and copy number variants (CNVs) calling. First, alignment of sequences against the $P$. avium genome was done using bwa-mem pipeline. Then, DELY and LUMPY were used for CNV detection and each.vcf file was validated using "vcfvalidator" tool of vcftools ${ }^{28}$. Intergation and filtering of DELLY and LUMPY output was realized using the "methodsMerge" command of the "intansv" package in
$\mathrm{R}$ with default parameters ${ }^{29}$. The $\mathrm{CNV}$ detection was performed by separating DNA sequences into fragments according to the sequencing depth of bases and the alignment results. Then, the $P$-value was calculated for each fragment to estimate its probability to be a $\mathrm{CNV}$. The fragments that passed the criteria (fragment length longer than $2 \mathrm{~kb}, p$-value $\leq 0.05$, and mean depth $<0.5$ or $>2.0$ ) were identified as CNVs.

\section{Linkage disequilibrium}

Potential linkage disequilibrium was detected by using PLINK. Pairwise $r^{2}$ was obtained for all markers within a $0.5 \mathrm{Mb}$ window and data were fitted using a local polynomial regression fitting (LOESS) model ${ }^{23}$ implemented in R (v. 3.3.3) ${ }^{24}$. Background linkage disequilibrium (BLD) was estimated by bootstrapping; 1000 replications were performed, and on each replication, $r^{2}$ was calculated among 1000 randomly selected SNPs. The BLD value was chosen as the upper value of the $95 \%$ confidence interval of the $r^{2}$ distribution.

LD decay was calculated for four groups of populations (a) the entire population of the 21 cultivars, (b) the 'Breeding line" group, (c) the 'Landrace' group and (d) the 'Modern cultivar' group.

\section{Genetic variation on genes related with traits of interest}

Based on the report of Sánchez-Pérez et al. $^{30}$, thirty three candidate genes involved in the regulation of flowering time were selected. Additionally, the VCF-BED intersect tool $^{31}$ was employed in order to retrieve the SNPs and InDels variations that were mapped to the 119 annotated disease resistance genes, as well as the 16 pathogenesis-related genes, which play pivotal roles in the defense reactions against pathogens in the reference genome. Genetic variability of the candidate genes was explored across the different accessions and the potential effect of the genetic changes was studied, using snpEff v.4. $3^{20}$, by annotating each SNP, based on their predicted effect on the candidate genes.

\section{Results and discussion}

\section{Variation in the sweet cherry genome}

Genome sequencing of the 21 sweet cherry genotypes yielded $204.26 \mathrm{~Gb}$ of high quality clean data with an average sequence depth of 33.91x and effective mapping depth, to the genomic reference sequence of 'Satonishiki' cultivar, between $22.21 \times$ to $36.62 \times$ (Supplementary Fig. 1, Supplementary File 2). These results suggest a wellcovered genome with higher sequence and mapping depth compared to similar studies ${ }^{32,33}$.

Paired-end sequencing reads were mapped to the genomic reference sequence of Prunus avium cv. 'Satonishiki $^{13}$ resulting in an average mapping depth of $35.51 \times$. Genome-wide variation including 1,880,922 
single-nucleotide polymorphisms (SNPs), 452,544 small insertions or deletions (indels), 5,677 copy number variations (CNVs) and 6607 presence absence variations (PAVs) across 21 sweet cherry genotypes, was identified (Table 1). About $26.15 \%$ of the total number of SNPs are located in intergenic regions and $12.13 \%$ in coding regions. SNPs in genic regions include 82,081 synonymous, 146,144 non-synonymous substitutions. Moreover, our analysis identified 411,957 intronic variants and 29,730 and 36,647 SNPs in $5^{\prime \prime}$ and $3^{\prime}$ UTRs, respectively (Fig. 1b). The cultivars 'Wild' and 'Mie' have the highest and lowest number of SNPs, respectively (Table 1). Due to self-incompatibility of this species, most of the SNPs/ InDels are heterozygous and the wild genotype possesses the greatest number of heterozygous SNPs (Fig. 2b). Most of the nucleotide changes can be classified as transitions $(11,759,094)$, with a transition/transversion ratio $(\mathrm{Ts} / \mathrm{Tv}$ ratio) of 1.4675 . Based on the type of change and its predicted effect, $0.36 \%$ of the SNPs were predicted to have a high impact (e.g., stop codon gaining, frameshift), 2.34\% a moderate (e.g., non-synonymous change, non-disruptive frameshift), and $1.56 \%$ a low impact (e.g., synonymous coding/start/stop, start gained) (Fig. 1b). These findings are in accordance with Shirasawa et al. ${ }^{13}$; their values were $0.7 \%, 6.4 \%$ and $4.6 \%$ for high, moderate and low impact mutations, respectively. The non-synonymous-tosynonymous substitution ratio $(\mathrm{dN} / \mathrm{dS})$ for the SNPs in the coding regions was 1.78 . For crops or species that are propagated clonally, synonymous SNPs are outnumbered by non-synonymous SNPs, while the opposite is more commonly met in wild species ${ }^{34}$. However, this value is much higher to the values reported for pigeon pea $\left(1.18^{35}\right)$, tomato $\left(1.23^{34}\right)$, peach $\left(1.06^{36}\right)$, Chinese plum $\left(1.30^{37}\right)$ and grapevine $\left(1.17^{32}\right)$. The higher $\mathrm{dN}$ frequency in our population of sweet cherry may possibly be the result of the artificial selection pressure imposed by breeding in combination with the vegetative propagation through grafting of domesticated cultivars.

We produced a unified catalog of SVs called by at least two of these four bioinformatics tools and these are described in Table 1. This catalog is comprised by 634 deletions, 192 insertions, 2312 duplications, inversions, or translocations (intra- or inter-chromosomal) and 5677 copy number variants (average length $5.2 \mathrm{~kb}$ ). The CNVs ranged from 4834 in cv. 'Petrokeraso Tragano Achaias' to 5963 in cv. 'Vasiliadi'. The distribution and corresponding annotations of PAVs and CNVs have been established (Supplementary Files 3 and 4). Shirasawa et al. ${ }^{13}$ WGRS study on six sweet cherry cultivars, has identified a lower number of sequence variants $(1,179,268)$, SNPs $(1,016,866)$ and insertions/deletions $(162,402)$, indicating higher genome wide variations in the present study, probably due to the larger number of genotypes.
Regarding InDels, a total of 427,160 variants were identified, of which 204,376 were insertions and 222,784 were deletions. Among those with potential functional consequences, $1.52 \%$ were located within gene exons and $0.2 \%$ in splice site regions, while $1.37 \%$ of Indels were located in $5^{\prime}$ - and 3'- UTR regions. The vast majority of the Indels was located upstream or downstream of genes and in intergenic regions. The size of insertions ranged from 1-29 nucleotides and deletions were in the range of 1-44 nucleotides in length. However, most of the insertions and deletions (25.2\%) were of a single nucleotide only. Di- and trinucleotide insertions and deletions accounted for $11.02 \%$ and $7.4 \%$ of the total InDels, respectively (Fig. $1 \mathrm{~b}$ and Supplementary File 5). Similar results have been observed by Varshney et al. ${ }^{38}$ in 429 chickpea accessions. Moreover, among larger InDels of $\geq 4$-nt, the total number of deletions was slightly higher than insertions (Fig. 1b). Similar to the SNP analysis, the "Wild" genotype demonstrated the greatest number and 'Mieza' the lowest number of InDels (Fig. 1a); wild genotype clearly presents the most diverse germplasm reservoir (Fig. 3a, b).

We further analyzed the distribution of so called largeeffect SNPs, which may potentially disable gene functions. It was found that within the 3187 SNPs in codon premature termination, 893 SNPs disrupt splicing donor or acceptor sites of the genome, 4211 SNPs are related to alteration of initiation methionine residues, and 571 SNPs replace terminators with curtain amino acid residues that lead to longer ORFs (Fig. 2a).

\section{Linkage disequilibrium (LD) analysis}

Detailed understanding of the linkage disequilibrium in a population of cultivars is crucial when considering the application of association genetics or GWAS in a species. LD is measured as the squared correlation coefficient $\left(r^{2}\right)$ between SNPs decays to $50 \%$ of its maximum at $5 \mathrm{~kb}$ and $90 \%$ of its maximum at $55 \mathrm{~kb}$ (Fig. 1c). The majority of SNP pairs with $r^{2}$ in near complete disequilibrium $(>0.8)$ are found at physical distances less than $10 \mathrm{~kb}$ (Supplementary Fig. 5). This relatively rapid decay of LD suggests that genome-wide association studies (GWAS) form a potential tool applicable for sweet cherry that will enable high-resolution mapping of genes associated with traits of agricultural significance. In comparison to pre-defined groups, we also found that LD decayed rapidly to an average $r^{2}$ of 0.8 within $20 \mathrm{~kb}$ for both modern cultivars and breeding lines (Fig. 1c). Moreover, the LD decay rate was faster in the modern cultivars than in the landraces suggesting a higher frequency of genetic recombination in the modern cultivars (Fig. 1c).

In Prunus, whole-genome diversity has been exploited using both SNPs and whole-genome resequencing data with a minor, or major, depth coverage ${ }^{39}$. Genome-wide data has identified fast LD decay in apricot, spanning 







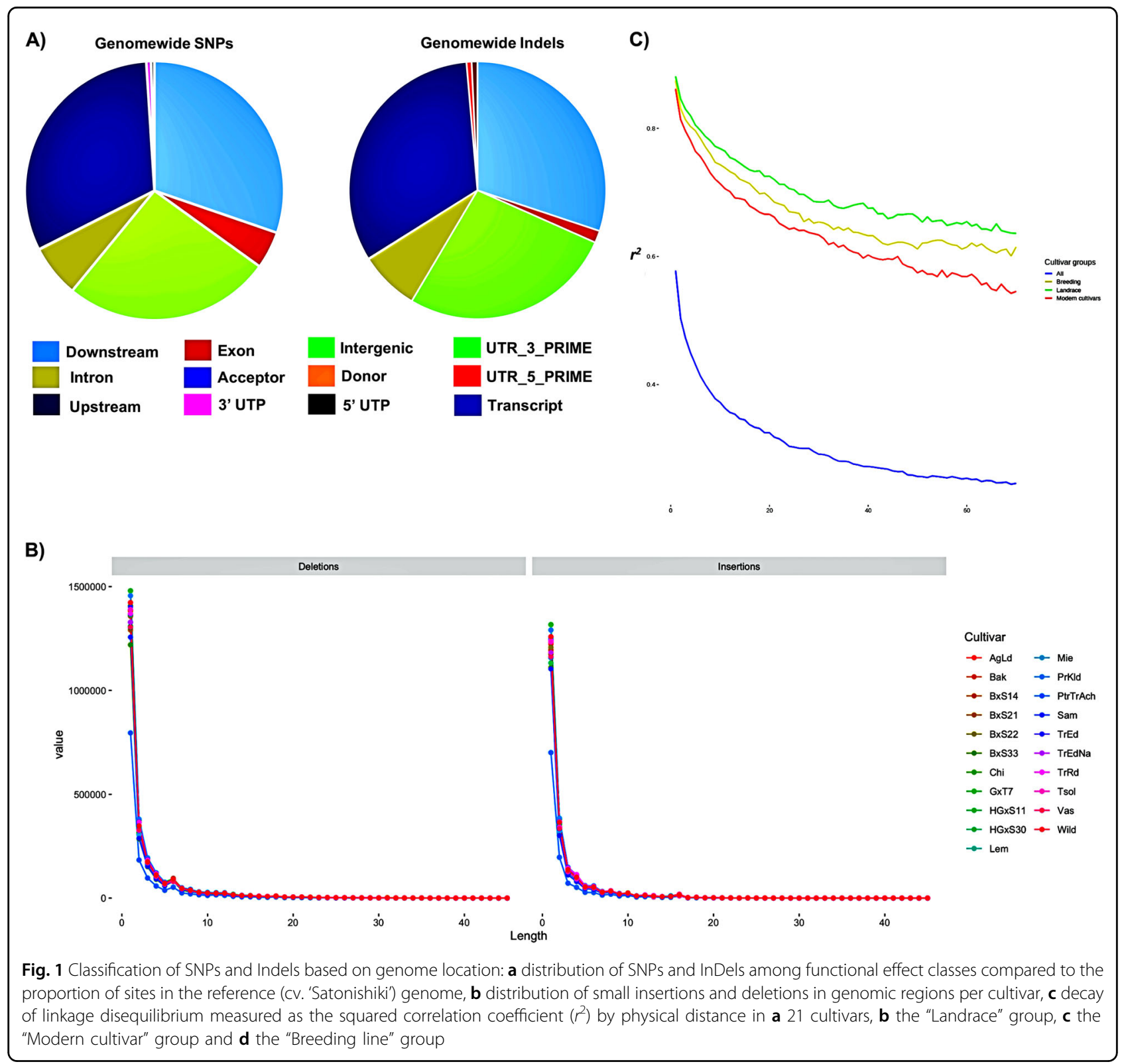

$<100 \mathrm{bp}^{40}$. Similarly, LD decays fast in ornamental $P$. mume accessions $\left(r^{2} \leq 0.2\right.$ at $50 \mathrm{~kb}$ to few hundreds of base-pairs depending on the subgroup ${ }^{37}$ and moderately in $P$. avium landraces and cultivars $\left(r^{2} \leq 0.2\right.$ at $\left.100 \mathrm{~kb}^{4}\right)$. The lower LD extensions oberved in sweet cherry $(P$. avium) compared to the LD observed in peach (P. persica) $\left(r^{2} \leq 0.2\right.$ between 0.8 and $1.4 \mathrm{Mb}$ depending on the population $^{41}$, are possibly related to the selfincompatibility system that was previously described in sweet cherry ${ }^{4}$.

\section{Population structure of sweet cherries}

The population structure of the Greek sweet cherry germplasm was studied by employing the whole genome sequencing data. The hierarchical clustering and neighborjoining (NJ) tree methods, group the genotypes according to their genealogy (Fig. 4a and Supplementary Fig. 2). Similarly to the NJ tree, PCA has separated the Greek genotypes into three main groups (Fig. 4b). Additionally, TrRd and TrEd are clustered, but their (short) distance does indicate some level of genetic divergence among them. Ganopoulos et al. ${ }^{2}$ reported similar clustering using SSR markers. The discriminant analysis of principal components (DAPC) with SNPs revealed a clear separation of distinct genetic clusters. Membership probabilities, interpreted as proximities of individuals to different clusters ${ }^{23}$, showed that genome-wide SNP markers achieved unambiguous separation of all groups (Fig. 4c). 

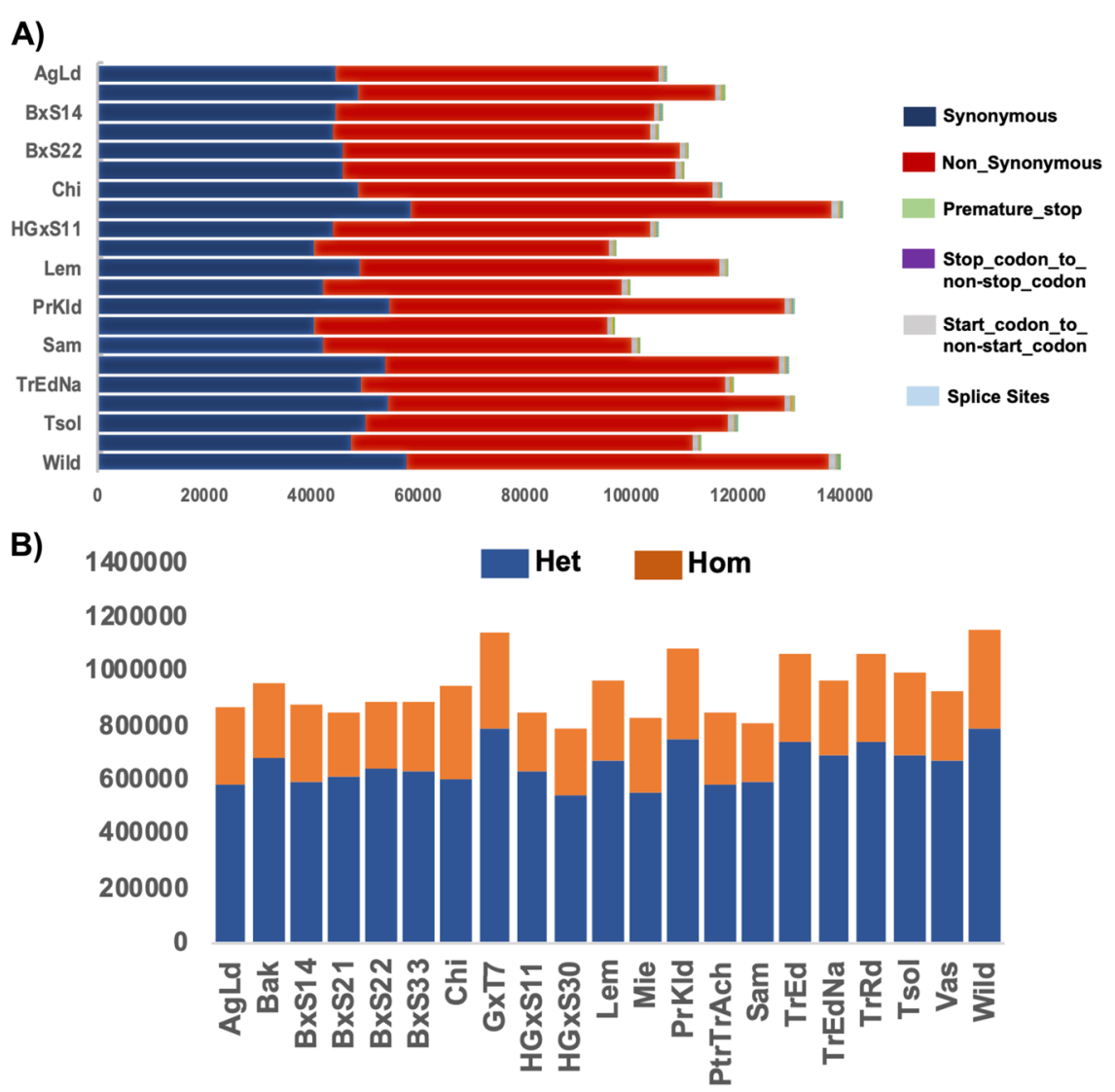

Fig. 2 a Number of large-effect SNPs in genome of 21 sweet cherry accessions. The $X$-axis displace the sample name, and $Y$-axis shows the number of each kind of large-effect SNP; $\mathbf{b}$ SNPs and InDels genetic diversity among 21 sweet cherry accessions. Het = Heterozygosity, Hom = Homozygosity

DAPC results presented a similar picture to that portrayed by PCA, but at a greater detail. Our germplasm collection appears to be separated into two clusters (Supplementary Fig. 3A). The optimal number of two clusters was also indicated by the Bayesian Information Criterion (BIC) statistic $(K=2)$ based on the Discriminant Analysis of Principal Components (DAPC) (Supplementary Fig. 3B). Cluster 1 (green) comprised of 11 accessions, most of them originating from the area of Naousa and four other accessions originating from the areas of Aridea, Chios island, Evia and Samos island. Cluster 2 (orange) includes a diverse group of accessions composed of 10 sweet cherry cultivars, mostly from the area of Edessa, and one wild cherry genotype.

We studied whether the groups defined a priori, represent statistically significant subpopulations by pairwise comparison of two measures of differentiation; Fst and Nei's standard genetic distance (Dst). The highest genetic distance and Fst values were observed between the breeding lines group and the wild species (Table 2). On the contrary, the minimum genetic distance was determined between landraces and modern cultivars suggesting a narrow genetic background of currently cultivated sweet cherry cultivars.

The wild progenitor of Greek cultivated sweet cherry cultivars, showed the highest nucleotide diversity $(2.31 \times$ $10^{-3}$ ) among the four predefined groups (Fig. 5). A very narrow domestication bottleneck was observed between the wild progenitor and landraces $(\pi$-wild/ $\pi$-landraces $=$ 1.46). This bottleneck is much weaker than the one observed in peach ${ }^{36}$ ( $\pi$-wild/ $\pi$-landraces $\left.=2.92\right)$, but still higher than other fruit-tree species such as grape ${ }^{42}$ and apple $^{43}$ that lack domestication bottlenecks, indicating an effect of the artificial selection on the sweet cherry genomes.

The "modern" $\left(\pi=1.47 \times 10^{-3}\right)$ and "breeding" $(\pi=$ $1.46 \times 10^{-3}$ ) cultivars share the same level of genetic diversity, suggesting a similar genetic background of the current cultivated sweet cherry cultivars, that retained the genetic diversity during their traits' improvement. However, the positive Tajima's D values (Supplementary File 7) and the limited genetic diversity and differentiation $(F s t=0.09)$ between the "modern" and "breeding cultivars" is a major constrain for further trait improvement, 


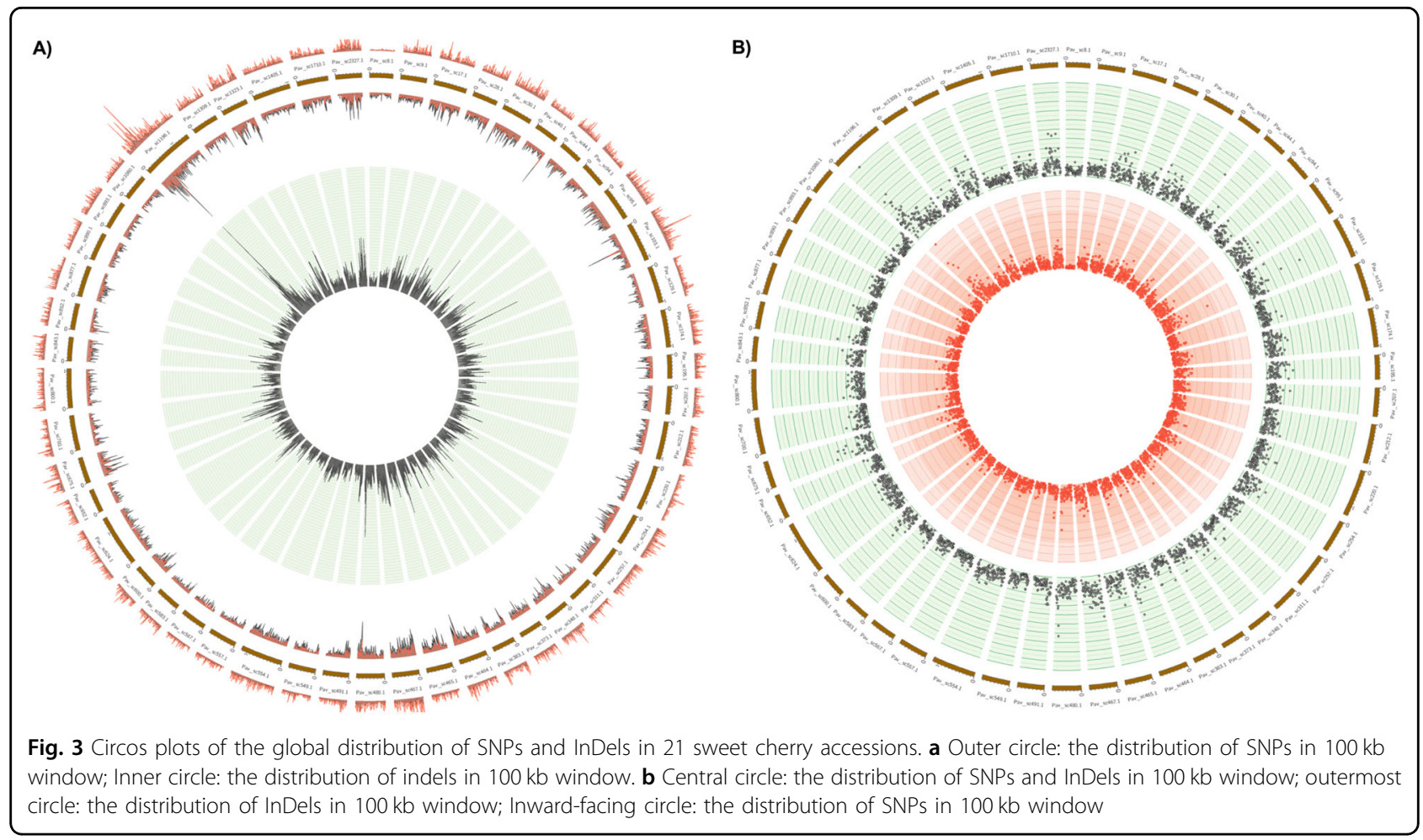

suggesting that future breeding programs need to introgress material from wild genotypes.

\section{Variation in genes involved in flowering time and dormancy}

A set of 13 candidate genes were selected, based on previous QTL studies, that are involved in flowering time and bud dormancy among various sweet cherry cultivars and other well-studied model plants such as Arabidopsis thaliana, i.e., Histone-lysine N-methyltransferase CLF (CLF), the Polycomb group (PcG) protein, the Embryonic Flower gene (EMF2), FLOWERING LOCUS C (FLC), MADS-box transcription factor SUPPRESSOR OF OVEREXPRESSION OF CO 1 (SOC1), FRIGIDA (FRI) and Far-red-impaired responsive protein (FAR1) ${ }^{39,44}$. The dataset of SNPs found in each gene is summarized in Supplementary File 6 and the mutations in the $5^{\prime}$ or $3^{\prime}$ UTR, exons and introns with high impact to the function, such as stop codon gain/loss, of the 13 candidate genes are reported on Fig. 6.

The majority of knowledge on the genetic basis of flowering time is obtained from studies on Arabidopsis thaliana (see ref. ${ }^{30}$ and references therein). More than 60 genes have been identified to control flowering time in Arabidopsis $^{45}$. Among them, SNPs present in genes including CONSTANS (CO), FLOWERING LOCUS C (FLC), VERNALIZATION INSENSITIVE 3, PHYTOCHROME D, GIBBERELLIN, etc. or coding region deletions as in FRIGIDA gene, result in significantly different phenotypes for flowering time. Dominant alleles of FRI generate late flowering phenotypes while FRI mutants are early flowering ${ }^{4-48}$ identified ParSOC1 as a candidate that could be involved in the regulation of dormancy break of vegetative shoots in apricot. Loss-of-function mutations in the EMF genes result in direct flowering in Arabidopsis, bypassing vegetative shoot growth ${ }^{49}$.

In the present study, no high-impact mutations were found in the flowering time $\mathrm{T}$ genes (FT), but some moderate variants in these genes (missense_variant; $p$. Ser175Tyr) were detected (Supplementary File 6).

Other high impact mutations affected genes associated with bud dormancy. A stop gained mutation specific of the only wild cherry genotype used, was found in the dormancy-associated MADS-box (DAM6) (Pavsc0000257.1). Dormancy-associated MADS-box (DAM) gene expression has been implicated in the establishment and maintenance of endodormancy. DAM6 genes are upregulated during growth cessation and downregulated by cold exposure during winter ${ }^{39}$. DNA methylations and small interfering RNAs are involved in the silencing of the sweet cherry PavMADS1 during cold accumulation and dormancy release ${ }^{50}$ with silencing of PavMADS1 and PavMADS2 coinciding with an increase in Flowering Locus T expression during dormancy in P. avium.

According to the results obtained from this analysis, some cultivars clustered according to their flowering time (Fig. 6). For instance all of the late-flowering cultivars 

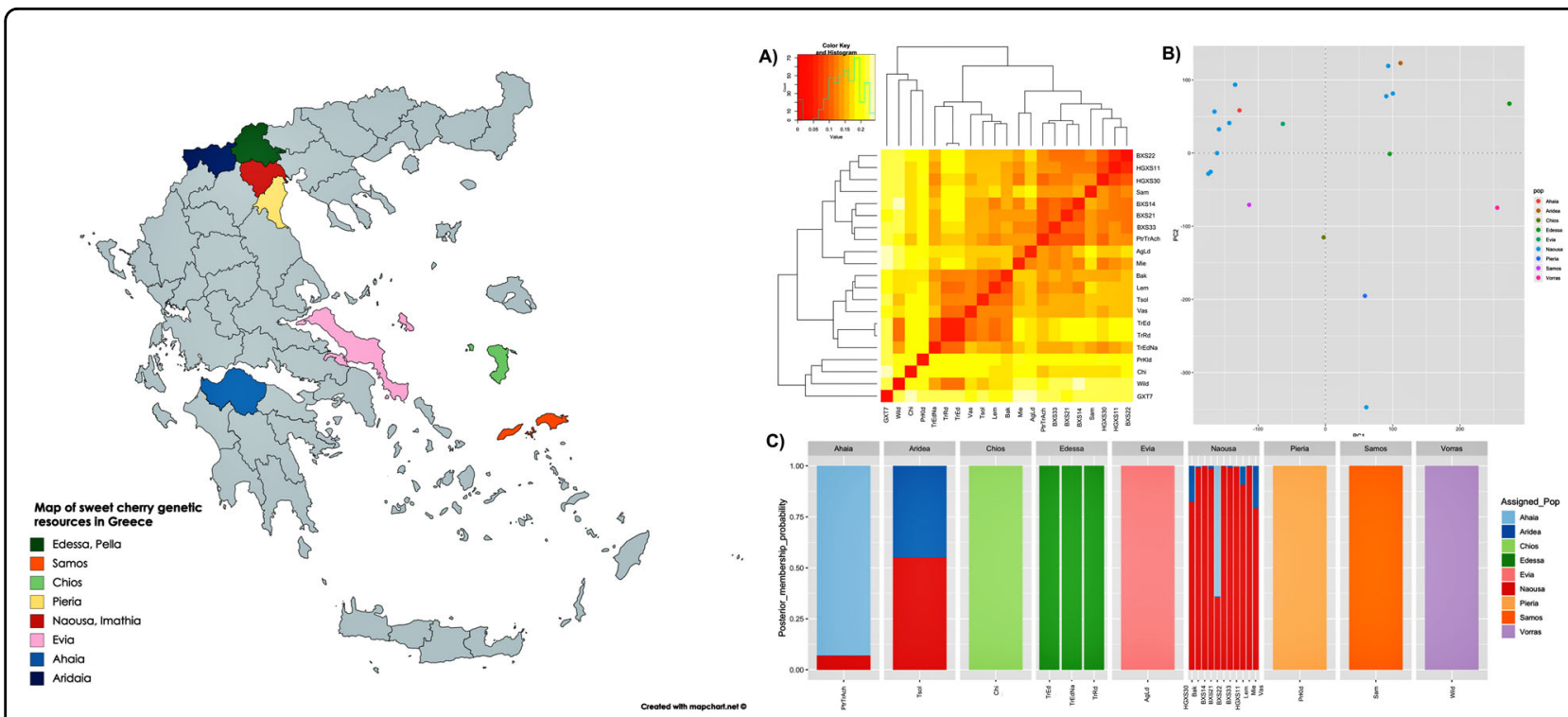

Fig. 4 Population structure in cultivated sweet cherries. a Heat map depicting genomic relationships between individual sweet cherry accessions lines. Individuals are across the $X$ - and $Y$-axis, with each square indicating the genomic relationship between the two respective individuals. The magnitude of the relationship is indicated by the color range (one being the relationship between an individual and itself, and 0 being the mean of the data set). $\mathbf{b}$ Principal component analysis (PCA) plot of the first two components of 21 cultivars using whole genome SNP data. PC1 and 2 axes account for 21.4 and $6.59 \%$ of the variation, respectively; c DAPC scatterplots and membership probabilities for SNP data. The scatterplots show the first two principal components of the DAPC. Geographic samples are represented in different colors with individuals shown as dots. Membership probabilities (in bar plots), interpreted as proximities of individuals to different clusters, show clear-cut separation of genetic groups for the SNP data

clustered together; while similar clustering was observed for most of the early-flowering cultivars. The subset of early-flowering cultivars that are clustered with those of late-flowering possibly, suggests that additional genes have an important impact on the flowering time of these sweet cherry cultivars. However, the observed genetic variation in flowering time related genes among the Greek sweet-cherry cultivars could be a valuable source of genetic markers for future breeding programs.

\section{Variation in genes involved in defense reactions against pathogens}

In this study, we selected a set of 119 defense-related genes (RPM1, RPP13, RGA2 homologs) which were previously predicted and being functionally annotated as disease resistance genes in the sweet cherry genome according to Shirasawa et al. ${ }^{13}$. These genes are usually clustered in close physical mapping and underlying QTLs have been previously described as involved in defense reactions against numerous plant pathogens ${ }^{51}$.

The SNPs and InDels variations observed according to our WGRS across the 21 sweet cherry accessions in each disease resistance gene are summarized in Supplementary Files 8 and 9. The total number of variants were 2468 and they were mapped on $107 R$ genes (almost $90 \%$ of the genes tested). The SNPs distribution (2241 in total) among the 21 genotypes and according to their annotation effect is depicted in Fig. 7a. The majority of them
Table 2 Genetic distances between the four predefined groups

\begin{tabular}{lllll} 
Predefined groups & Landraces & $\begin{array}{l}\text { Modern } \\
\text { cultivars }\end{array}$ & Breeding lines Wild \\
\hline Landraces & 0 & & & \\
Modern cultivars & 0.018 & 0 & 0 & 0 \\
Breeding lines & 0.029 & 0.094 & 0 & 0.2 \\
Wild & 0.037 & 0.13 & 0.2 & 0 \\
\hline
\end{tabular}

Pairwise estimates of Nei's standard genetic distance (Dst) between the predefined groups. Color scale: Red $=0$ to Blue $=1$.

(1188 SNPs) was heterozygous and were missense variants. Forty-four $R$ NBS-LRR genes, have stop-codon gain and loss SNP mutations in the coding sequences (CDS) with high impact (Supplementary File 8). A total number of 227 InDels and their effect were identified (Fig. 7b; Supplementary File 10). The insertions and deletions length were up to 41 and $91 \mathrm{bp}$, respectively. More than $42 \%$ (97 InDels) were of high impact referring mainly mutations of frameshift variant annotations effects. The genotypes "Trrd", "Tred", "Tredna" and "Wild" demonstrated the largest number of high impact SNPs and InDels variations, among the 21 sweet cherry genotypes (Fig. 7).

In order to have a more deciphering view, the 21 sweet cherry genotypes were hierarchically clustered by using all 
the high impact mutations, both SNPs and InDels, in the 57 NLR genes (Fig. 8). The "Tredna" cultivar was found to have the most abundant high impact variants. The "Wild" and "Gxt7" genotypes were clustered together with "Tred" and "Trrd". Among the 57 genes which contributed mostly in the variants with high impact

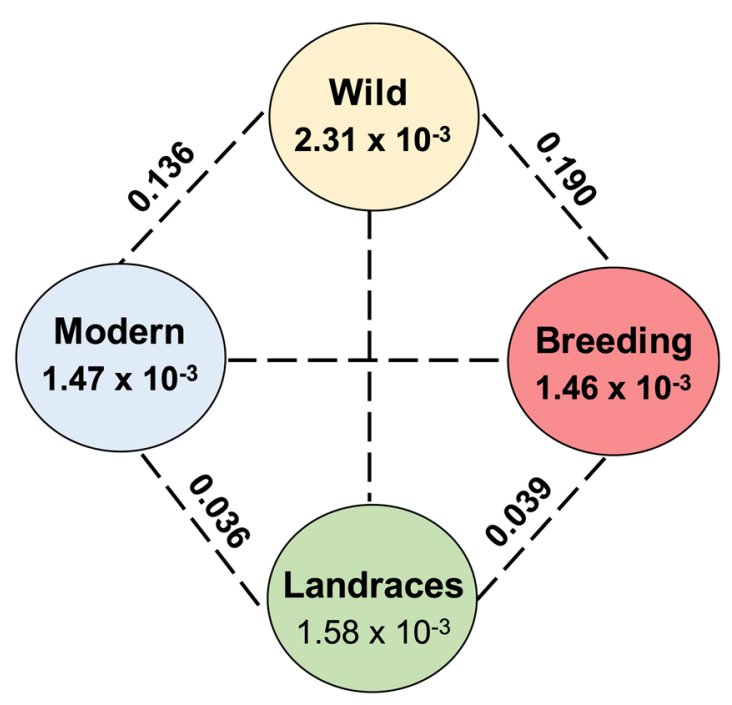

Fig. 5 Nucleotide diversity $(\pi)$ and population divergence (Fst) across the four groups. Values between pairs (dashed-line) indicate population divergence (Fst) and value in each circle represents nucleotide diversity $(\pi)$ for the group
(Pav_sc0000136.1_g230.1.br, Pav_sc0000387.1_g020.1.br, Pav_sc0006454.1_g020.1.br), the first two contain NBARC domains, whereas Pav_sc0006454.1_g020.1.br contains two copies of LRRs of type LRR_4. These genes are homologs of RPM1 which is a quite dynamical polymorphic locus in Arabidopsis ${ }^{52}$. More recently, an evolutionary analysis in sweet cherry genome revealed also that diversifying episodes acting on the NB-ARC domains of Resistance Gene Analogs (RGAs) occurred putatively affecting their ligand-binding specificities through positive selection ${ }^{3}$. Thus, the results of this WGRS analysis, that showed variation in highly abundant SNPs and InDels occuring in NLR genes confirm that these NLR receptors are prone to variation among the sweet cherry accessions. All these findings allow us to assign these NSB-LRR proteins as the foremost surveillance mechanism against rapidly evolving pathogens, and providing breeders with effective genomics tools to speed-up the development of sweet cherry varieties with more durable resistance.

We also selected the functionally annotated PATHOGENESIS-RELATED (PR) proteins which encode plant species-specific genes induced in response to infection with fungi, bacteria or viruses in order to estimate the variation upon them across the 21 accessions. These genes have been associated with basic-scale defenses through coordinated interactions within signaling pathways leading at the establishment of systemic acquired resistance (SAR) as well as induced systemic



Fig. 6 Twenty-four SNPs with high impact alterations identified in genes involved in flowering time and dormancy 


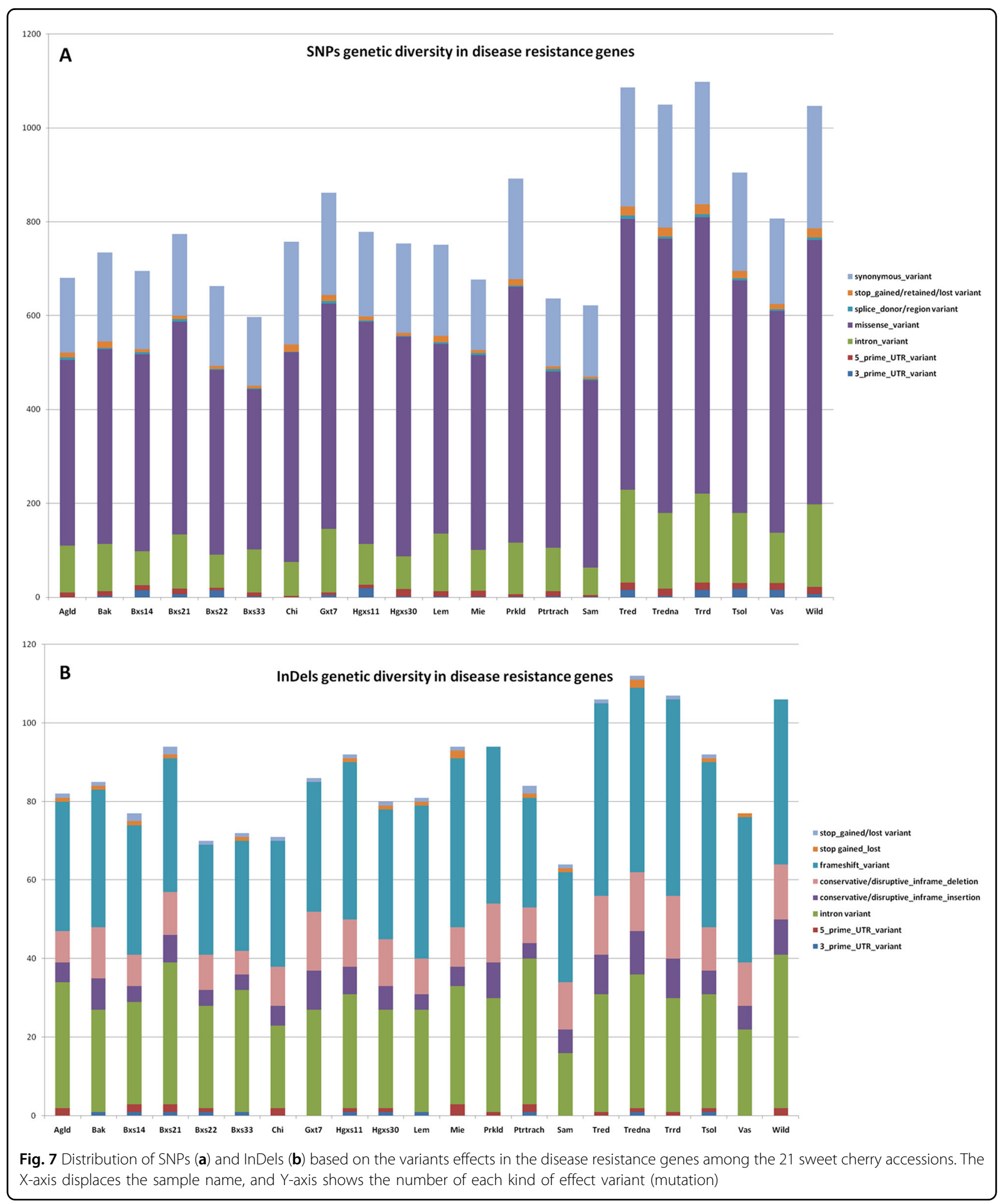

resistance (ISR) $)^{53}$. Most of the $P R$ genes in sweet cherry genome belong to PR-1 and PR-4 families.

Our results showed that 65 SNPs and 20 InDels variations was observed among the $P R$ genes across the sweet cherry accessions (Supplementary Files 10 and 11). These variations were found to disperse across nine out of the $16 P R$ genes in the genome. The majority of them ( 25 and 15 , respectivelly) were found to map in two genes (Pav_sc0000029.1_g200.1. 

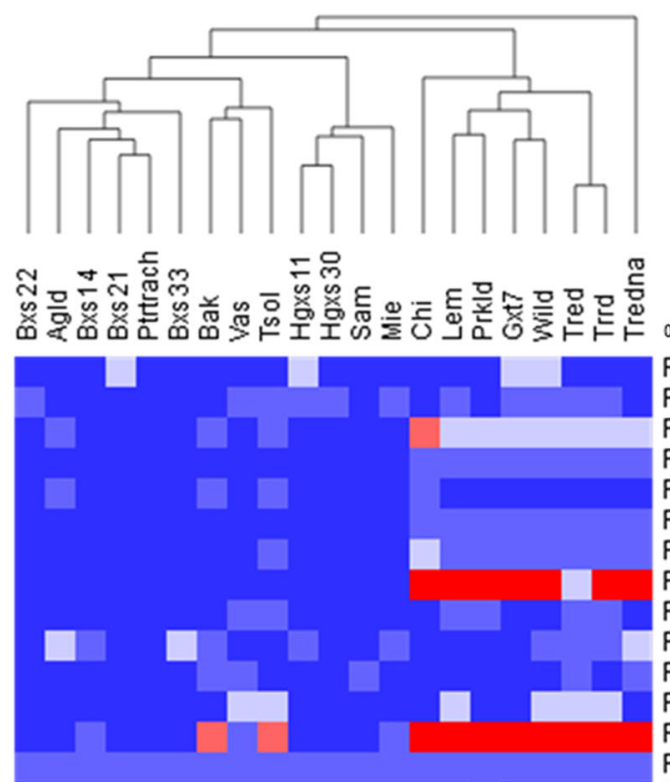
$5.00 \quad 5.00 \quad 10.00$

Pav_sc0000095.1_g1050.1.mk Pav_sc0000099.1_g290.1.mk Pav_sc0000136.1_g120.1.br Pav_sc0000136.1_g180.1.br Pav_sc0000136.1 g190.1.br Pav_sc0000136.1_g200.1.br Pav_sc0000136.1 g220.1.br Pav_sc0000136.1_g230.1.br Pav_sc0000182.1_g010.1.br av_sc0000257.1_g1010.1.br Pav_sc0000379.1_g160.1.mk Pav_sc0000387.1 g010.1.br Pav_sc0000387.1_g020.1.br Pav_sc0000387.1 g040.1.br Pav_sc0000428.1_g070.1.mk Pav_sc0000549.1_g250.1.br Pav sc0000608.1 g100.1.br Pav_sc0000613.1_g070.1.br Pav_sc0000647.1 g010.1.mk Pav_sc0000647.1_g030.1.br Pav_sc0000713.1_g060.1.br Pav_sc0000717.1_g010.1.br Pav_sc0000878.1_g070.1.mk Pav sc0001065.1 g050.1.br Pav_sc0001097.1_g070.1.br Pav_sc0001282.1_g060.1.mk Pav_sc0001367.1_g020.1.br Pav_sc0001369.1_g400.1.mk Pav sc0001412.1 g070.1.br Pav_sc0001520.1_g090.1.br Pav_sc0001524.1_g050.1.mk Pav_sc0001718.1_g070.1.mk Pav_sc0001718.1_g150.1.mk Pav_sc0001721.1_g010.1.br Pav_sc0001985.1_g030.1.mk Pav_sc0001985.1_g060.1.br Pav_sc0002033.1_g010.1.br Pav sc0002237.1 g030.1.br Pav_sc0002237.1_g040.1.br Pav_sc0002684.1_g030.1.br Pav_sc0003261.1_g170.1.br Pav_sc0003823.1_g140.1.br Pav_sc0004383.1 g140.1.br Pav_sc0004383.1_g150.1.br Pav_sc0005121.1_g040.1.br Pav_sc0005463.1_g010.1.br Pav_sc0006272.1_g050.1.br Pav_sc0006420.1 g020.1.br Pav_sc0006454.1_g020.1.br Pav sc0009154.1 g020.1.br Pav_sc0009370.1_g040.1.br Pav_sc0009370.1_g050.1.br Pav_sc0009370.1_g060.1.br Pav_sc0010906.1_g060.1.br Pav_sc0012624.1 g010.1.br Pav_c04046203.1_g010.1.mk Pav_c04078237.1_g010.1.br

Fig. 8 Hierarchical clustering heatmap depicting genomic relationships between individual sweet cherry accessions for the total number of SNPs and InDels with high impact mutations identified and mapped in 57 NLR genes involved in defense reactions against pathogens. Sweet cherry accessions and NLR genes are across the $\mathrm{X}$ - axis and Y-axis, respectively. The magnitude and the color range of the relationship are indicated by the total number of variations across each sweet cherry accession for each NLS gene 
mk and Pav_sc0000030.1_g1270.1.mk spanning scaffolds Pav_sc0000029.1 and Pav_sc0000030.1, respectively).

The majority of these 65 SNPs were intron variants (46 in their number), eight were $3^{\prime}$ and $5^{\prime}$ prime UTR variants and 11 were synonymous, missense and splice-region variants (Supplementary Files 10 and 11). The highest number of variations were found in Lem (58), Trrd (56), while the lowest in Hgxs11 (25) genome accession. The majority of InDels mutations were of intron variant effect with no high impact.

Overall, as it was expected, we found fewer variations among the 21 sweet cherry accessions for $P R$ genes in comparison with the disease resistance $N L R$ receptors (NBS-LRR-containing genes), as $P R$ genes comprise the basal defense and they are more conservative in structure, under rather purifying selection and less abundant in numbers across plant genomes. Therefore, our results indicate that $N L R$ genes are promising resources for breeding broad-spectrum resistance as it was previously also mentioned ${ }^{54,55}$.

\section{Conclusion}

This is the first report of whole genome genetic variation characterization between sweet cherry cultivars. By WGRS we have captured the majority of genetic variation (>95\%) of Greece's cultivated germplasm. A high degree of heterozygosity and potentially functional variation was revealed, as indicated by the high nonsynonymous-tosynonymous substitution ratio. Most of the genotypes were clustered according to their geographic region of origin, with some exceptions, which indicates potential movement of the germplasm across regions.

The evaluation at whole genome level of cultivated and wild germplasm is important for the identification of allelic variations with phenotypic effects. We have discovered numerous high impact allelic variants on flowering and diseases resistance genes. Further characterization of the precise impact of such variants on flowering time and defense reactions against pathogens will enable their implementation in molecular breeding programs to fine tune flowering, maturity time and diseases resistance in cherry cultivars. Hence, our study has established the foundation for further large scale characterization of sweet cherry germplasm, aiming at enabling breeders to use diverse germplasm and allelic variants towards developing improved cherry varieties with increased productivity.

\section{Acknowledgements}

This project has received funding from the Hellenic Foundation for Research and Innovation (HFRI) and the General Secretariat for Research and Technology (GSRT), under grant agreement No. 148.

\section{Author details}

${ }^{1}$ Laboratory of Pomology, Department of Agriculture, Aristotle University of Thessaloniki, 54124 Thessaloniki, Greece. ${ }^{2}$ Institute of Plant Breeding and
Genetic Resources, ELGO-DEMETER. Thermi, Thessaloniki 570001, Greece. ${ }^{3}$ Perrotis College, American Farm School, Thessaloniki GR-57001, Greece. ${ }^{4}$ Laboratory of Forest Genetics \& Tree Breeding, Faculty of Agriculture, Forestry \& Environmental Science, Aristotle University of Thessaloniki, Thessaloniki, Greece. ${ }^{5}$ Institute of Plant Breeding and Genetic Resources, ELGO-DEMETER. Department of Deciduous Fruit Growing, Naoussa 59035, Greece. ${ }^{6}$ Institute of Applied Biosciences, CERTH, Thermi, Thessaloniki 570 01, Greece

\section{Data availability}

All of the raw reads of the sweet cherry accessions generated in this study have been deposited in the public database of National Center of Biotechnology Information under SUB6334624.

\section{Conflict of interest}

The authors declare that they have no conflict of interest.

Supplementary Information accompanies this paper at (https://doi.org/ 10.1038/s41438-020-0281-9).

Received: 11 November 2019 Revised: 17 January 2020 Accepted: 18 February 2020

Published online: 01 May 2020

\section{References}

1. McCune, L. M., Kubota, C., Stendell-Hollis, N. R. \& Thomson, C. A. Cherries and health: a review. Crit. Rev. Food Sci. Nutr. 51, 1-12 (2010).

2. Ganopoulos, I. V., Kazantzis, K., Chatzicharisis, I., Karayiannis, I. \& Tsaftaris, A. S. Genetic diversity, structure and fruit trait associations in Greek sweet cherry cultivars using microsatellite based (SSR/ISSR) and morpho-physiological markers. Euphytica 181, 237-251 (2011).

3. Zambounis, A. et al. Evidence of extensive positive selection acting on cherry ('Prunus avium'L.) resistance gene analogs (RGAs). Aust. J. Crop Sci. 10, 1324 (2016).

4. Campoy, J. A. et al. Genetic diversity, linkage disequilibrium, population structure and construction of a core collection of Prunus avium L. landraces and bred cultivars. BMC Plant Biol. 16, 49 (2016).

5. Ganopoulos, I. et al. A mini review on morphological and genetic diversity of sweet (Prunus avium L.) and sour cherry (Prunus cerasus L.) cultivars. (Nova Science Publishers, 2016).

6. Peace, C. et al. Development and evaluation of a genome-wide 6K SNP array for diploid sweet cherry and tetraploid sour cherry. PLOS ONE 7, e48305 (2012).

7. Rawat, N. et al. Genome resequencing and transcriptome profiling reveal structural diversity and expression patterns of constitutive disease resistance genes in Huanglongbing-tolerant Poncirus trifoliata and its hybrids. Hortic. Res. 4, 17064 (2017).

8. Fernandez i Marti, A., Saski, C. A., Manganaris, G. A., Gasic, K. \& Crisosto, C. H. Genomic sequencing of Japanese plum (Prunus salicina Lindl.) mutants provides a new model for Rosaceae fruit ripening studies. Front. Plant Sci. 9, 21 (2018).

9. Zhang, J. et al. Genome-Wide Discovery of DNA Polymorphisms in Me (Prunus mume Sieb. et Zucc.), an ornamental woody plant, with contrasting tree architecture and their functional relevance for weeping trait. Plant Mol. Biol. Report. 35, 37-46 (2017)

10. Cao, K. et al. Comparative population genomics reveals the domestication history of the peach, Prunus persica, and human influences on perennial fruit crops. Genome Biol. 15, 415 (2014).

11. $\mathrm{Xu}, \mathrm{X}$. et al. Resequencing 50 accessions of cultivated and wild rice yields markers for identifying agronomically important genes. Nat. Biotechnol. $\mathbf{3 0}$ 105 (2012).

12. McClure, K. A., Sawler, J., Gardner, K. M., Money, D. \& Myles, S. Genomics: a potential panacea for the perennial problem. Am. J. Bot. 101, 1780-1790 (2014).

13. Shirasawa, K. et al. The genome sequence of sweet cherry (Prunus avium) for use in genomics-assisted breeding. DNA Res. 24, 499-508 (2017).

14. Yan, M., Zhang, X., Zhao, X. \& Yuan, Z. The complete mitochondrial genome sequence of sweet cherry (Prunus avium cv'summit'). Mitochondrial DNA 4, 1996-1997 (2019). 
15. Chen, T. et al. Characterization of complete chloroplast genome and phylogenetic analysis of sweet cherry Cerasus avium (L.) Moench (Prunoideae, Rosaceae). Mitochondrial DNA 3, 1274-1275 (2018)

16. Ono, K., Akagi, T., Morimoto, T., Wünsch, A. \& Tao, R. Genome re-sequencing of diverse sweet cherry (Prunus avium) individuals reveals a modifier gene mutation conferring pollen-part self-compatibility. Plant Cell Physiol. 59, 1265-1275 (2018)

17. Ganopoulos, I. et al. Towards sweet cherry (Prunus avium L.) breeding: phenotyping evaluation of newly developed hybrids. Euphytica 214, 99 (2018).

18. Li, H. \& Durbin, R. Fast and accurate long-read alignment with Burrows-Wheeler transform. Bioinformatics 26, 589-595 (2010).

19. DePristo, M. A. et al. A framework for variation discovery and genotyping using next-generation DNA sequencing data. Nat. Genet. 43, 491 (2011).

20. Cingolani, P. et al. A program for annotating and predicting the effects of single nucleotide polymorphisms, SnpEff: SNPs in the genome of Drosophila melanogaster strain w1118; iso-2; iso-3. Fly 6, 80-92 (2012).

21. Pembleton, L. W., Cogan, N. O. I. \& Forster, J. W. St AMPP: an R package for calculation of genetic differentiation and structure of mixed-ploidy level populations. Mol. Ecol. Resour. 13, 946-952 (2013).

22. Paradis, E., Claude, J. \& Strimmer, K. APE: analyses of phylogenetics and evolution in R language. Bioinformatics 20, 289-290 (2004).

23. Jombart, T., Devillard, S. \& Balloux, F. Discriminant analysis of principal components: a new method for the analysis of genetically structured populations. BMC Genet. 11, 94 (2010).

24. Murtagh, F. \& Legendre, P. Ward's hierarchical agglomerative clustering method: which algorithms implement Ward's criterion? J. Classif. 31, 274-295 (2014).

25. Zheng, L.-Y. et al. Genome-wide patterns of genetic variation in sweet and grain sorghum (Sorghum bicolor). Genome Biol. 12, R114 (2011).

26. Rausch, T. et al. DELLY: structural variant discovery by integrated paired-end and split-read analysis. Bioinformatics 28, i333-i339 (2012).

27. Layer, R. M., Chiang, C., Quinlan, A. R. \& Hall, I. M. LUMPY: a probabilistic framework for structural variant discovery. Genome Biol. 15, R84 (2014).

28. Danecek, P. et al. The variant call format and VCFtools. Bioinformatics 27, 2156-2158 (2011)

29. Yao, W. intansv: Integrative analysis of structural variations. $R$ package version 1.92 (2015).

30. Sánchez-Pérez, R., Del Cueto, J., Dicenta, F. \& Martínez-Gómez, P. Recent advancements to study flowering time in almond and other Prunus species. Front. Plant Sci. 5, 334 (2014).

31. Quinlan, A. R. \& Hall, I. M. BEDTools: a flexible suite of utilities for comparing genomic features. Bioinformatics 26, 841-842 (2010).

32. Liang, Z. et al. Whole-genome resequencing of 472 Vitis accessions for grapevine diversity and demographic history analyses. Nat. Commun. 10, 1190 (2019).

33. Hazzouri, K. M. et al. Whole genome re-sequencing of date palms yields insights into diversification of a fruit tree crop. Nat. Commun. 6, 8824 (2015).

34. Tomato Genome Sequencing, $C$. et al. Exploring genetic variation in the tomato (Solanum section Lycopersicon) clade by whole-genome sequencing. Plant J. 80, 136-148 (2014)

35. Varshney, R. K. et al. Whole-genome resequencing of 292 pigeonpea accessions identifies genomic regions associated with domestication and agronomic traits. Nat. Genet. 49, 1082 (2017).
36. Li, Y. et al. Genomic analyses of an extensive collection of wild and cultivated accessions provide new insights into peach breeding history. Genome Biol. 20, 36 (2019).

37. Zhang, Q. et al. The genetic architecture of floral traits in the woody plant Prunus mume. Nat. Commun. 9, 1702 (2018).

38. Varshney, R. K. et al. Resequencing of 429 chickpea accessions from 45 countries provides insights into genome diversity, domestication and agronomic traits. Nat. Genet. 51, 857 (2019).

39. Aranzana, M. J. et al. Prunus genetics and applications after de novo genome sequencing: achievements and prospects. Hortic. Res. 6, 58 (2019).

40. Mariette, S. et al. Genome-wide association links candidate genes to resistance to Plum Pox Virus in apricot (Prunus armeniaca). New Phytol. 209, 773-784 (2016).

41. Micheletti, D. et al. Whole-genome analysis of diversity and SNP-major gene association in peach germplasm. PLOS ONE 10, e0136803 (2015).

42. Zhou, Y., Massonnet, M., Sanjak, J. S., Cantu, D. \& Gaut, B. S. Evolutionary genomics of grape (Vitis vinifera ssp. vinifera) domestication. Proc. Natl Acad. Sci. USA 114, 11715-11720 (2017).

43. Duan, N. et al. Genome re-sequencing reveals the history of apple and supports a two-stage model for fruit enlargement. Nat. Commun. 8, 249 (2017).

44. Putterill, J., Laurie, R. \& Macknight, R. It's time to flower: the genetic control of flowering time. Bioessays 26, 363-373 (2004).

45. Ehrenreich, I. M. et al. Candidate gene association mapping of Arabidopsis flowering time. Genetics 183, 325-335 (2009).

46. Johanson, U. et al. Molecular analysis of FRIGIDA, a major determinant of natural variation in Arabidopsis flowering time. Science 290, 344-347 (2000).

47. Jung, C. \& Müller, A. E. Flowering time control and applications in plant breeding. Trends Plant Sci. 14, 563-573 (2009).

48. Trainin, T., Bar-Ya'akov, I. \& Holland, D. ParSOC1, a MADS-box gene closely related to Arabidopsis AGL20/SOC1, is expressed in apricot leaves in a diurnal manner and is linked with chilling requirements for dormancy break. Tree Genet. Genomes 9, 753-766 (2013).

49. Yoshida, N. et al. EMBRYONIC FLOWER2, a novel polycomb group protein homolog, mediates shoot development and flowering in Arabidopsis. Plant Cell 13, 2471-2481 (2001).

50. Rothkegel, K. et al. DNA methylation and small interference RNAs participate in the regulation of MADS-box genes involved in dormancy in sweet cherry (Prunus avium L.). Tree Physiol. 37, 1739-1751 (2017).

51. Larkan, N. J. et al. Multi-environment QTL studies suggest a role for cysteinerich protein kinase genes in quantitative resistance to blackleg disease in Brassica napus. BMC Plant Biol. 16, 183 (2016).

52. Tornero, P., Chao, R. A., Luthin, W. N., Goff, S. A. \& Dangl, J. L. Large-scale structure-function analysis of the Arabidopsis RPM1 disease resistance protein Plant Cell 14, 435-450 (2002).

53. El-Kereamy, A. et al. Prunus domestica pathogenesis-related protein-5 activates the defense response pathway and enhances the resistance to fungal infection. PLOS ONE 6, e17973 (2011).

54. Li, Z. et al. Overexpression of Arabidopsis nucleotide-binding and leucine-rich repeat genes RPS2 and RPM1 (D505V) confers broad-spectrum disease resistance in rice. Front. Plant Sci. 10, 417 (2019).

55. Wang, R. et al. Immunity to rice blast disease by suppression of effectortriggered necrosis. Curr. Biol. 26, 2399-2411 (2016) 